\title{
The Superfield Quantisation of a Superparticle Action with an Extended Line Element
}

\author{
P.L.FitzGerald \\ School of Computing and Mathematics, The Nottingham Trent University, Burton Street \\ Nottingham, NG1 $4 \mathrm{BU}, \mathrm{UK}$. \\ Peter.Fitzgerald@ntu.ac.uk \\ Received (Day Month Year) \\ Revised (Day Month Year)
}

\begin{abstract}
A massive superparticle action based on the generalised line element in $\mathrm{N}=1$ global superspace is quantised canonically. A previous method of quantising this action, based on a Fock space analysis, showed that states existed in three supersymmetric multiplets, each of a different mass. The quantisation procedure presented uses the single first class constraint as an operator condition on a general $\mathrm{N}=1$ superwavefunction. The constraint produces coupled equations of motion for the component wavefunctions. Transformations of the component wavefunctions are derived that decouple the equations of motion and partition the resulting wavefunctions into three separate supermultiplets. Unlike previous quantisations of superparticle actions in $\mathrm{N}=1$ global superspace, the spinor wavefunctions satisfy the Dirac equation and the vector wavefunctions satisfy the Proca equation.

The off-shell closure of the commutators of the supersymmetry transformations, that include mass parameters, are derived by the introduction of auxiliary wavefunctions.

To avoid the ghosts arising in a previous Fock space quantisation an alternative conjugation is used in the definition of the current, based on a Krein space approach.
\end{abstract}

Keywords: Superparticle; Supersymmetry.

\section{Introduction}

The dynamics for a supersymmetric relativistic point particle can be derived from an action that is proportional to the length of the worldline in super-Poincare spacetime $^{1}$. The Hamiltonian analysis of the simplest superparticle action with $\mathrm{N}=1$ supersymmetry $^{1}$, reveals one first class constraint, the mass shell constraint, and a second class constraint consisting of one four-component Majorana spinor. On canonical quantisation, the first class constraint becomes an operator condition on the superwavefunction. The second class constraints complicate the quantisation, requiring the solution of the Dirac brackets ${ }^{2}$. The result is a chiral superwavefunction containing component wavefunctions that obey the Klein-Gordon equation. In superfield theory, the chiral multiplet, composed of two complex scalars and a complex Majorana spinor, is used in the Wess-Zumino model ${ }^{3}$. The Wess-Zumino model defines an action from which the spinor wavefunctions are found to obey the Dirac equation. However, the Dirac equation is not a direct consequence of the solution 
of the canonical constraints that arise from these super-Poincare invariant actions. One aim of this paper is to derive the Dirac equation and higher spin wave equations directly from the constraints arising from a super-Poincare invariant action.

In a previous attempt to obtain the Dirac equation from quantisation of a superparticle action ${ }^{4}$ a more general $\mathrm{N}=1$ supersymmetric line element was investigated ${ }^{5}$ by Volkov and Pashnev. The extended line element had an additional term quadratic in the spinorial coordinates and is based on the most general form of the metric in $\mathrm{N}=1$ superspace ${ }^{6}$. Inclusion of the new term in the line element avoided the second class constraints that arise in other $\mathrm{N}=1$ superparticle actions ${ }^{1,7,8}$ and therefore simplified the quantisation. The model had one first class constraint that was quadratic in fermionic as well as bosonic momenta. The fermionic momenta only appear as part of supersymmetric covariant derivatives. To quantise the theory ${ }^{5}$, a Fock space was constructed using a Clifford algebra of fermionic annihilation and creation operators based on the supersymmetry generators and the supersymmetric covariant derivatives. However, this approach is not suitable to derive wave equations and the Dirac equation was not found for the spinor wavefunctions ${ }^{4}$. A further problem, in this approach, was that the Fock space states that formed a vector multiplet had negative norm ${ }^{5}$. A second aim of this paper is therefore to present an alternative method of quantisation the extended superparticle action ${ }^{5}$, in which there is a physical interpretation of all the particle states such that ghosts states are absent.

The additional term in superparticle worldline contains a second mass parameter that has interesting consequences in the quantisation. Instead of a single massive supersymmetric multiplet, that arises in the quantisation of the restricted worldline action, three supersymmetric multiplets were produced (two scalar multiplets and one vector multiplet), each with a different mass ${ }^{5}$. This may be of interest in the construction of models for particle physics, if the ghost states can be removed. In superparticle quantisations based on restricted worldline actions, such as the original $\mathrm{N}=1$ model $^{1}$ or the $\mathrm{N}=2$ superparticle with central charges that produces the hypermultiplet ${ }^{4,7,8,9}$, all the component wavefunctions have the same mass equal to the mass parameter in the superparticle action.

The method presented here uses the first class constraint as an operator condition $^{7,8}$ on a general $\mathrm{N}=1$ scalar superwavefunction in order to derive onand off-shell wavefunctions that form supersymmetric representations ${ }^{3,10,11}$. This alternative method is used to demonstrate that the extended superparticle action provides a valid model for supersymmetric quantum mechanics.

Sections 2 and 3 describe the superparticle action and Hamiltonian constraint. In Sections 4 and 5 the canonical quantisation procedure is detailed. The action of the operator constraint produces wave equations for each component wavefunction in the superfield. The initial wave equations derived from the quantised constraint are of second order in the spacetime derivatives but relate more than one component wavefunction i.e. the wave equations are not diagonal and mix the component wavefunctions.

However, in Section 6, transformations are derived from the original component 
wavefunctions (OCWFs) to a new set of transformed component wavefunctions (TCWFs) that diagonalise the wave equations. The TCWFs are found to satisfy the Klein-Gordon, Dirac and Proca equations for massive scalar, spinor and vector particles. The presence of the two mass parameters in the transformation on the wave equations produces three separate on-shell superfield multiplets that satisfy wave equations of differing mass. The three masses are related by a simple algebraic formula discovered by Volkov and Pashnev ${ }^{5}$.

In Sections 7, an action is derived for the OCWFs from which the original wave equations can be calculated. The action is invariant to global supersymmetry transformations. The variables in the action are the OCWFs and the conjugates of the OCWFs. The form of the conjugation used is decided in subsequent sections based on the demand that the terms from all the TCWF supermultiplets contribute to the quantum probability with the same sign. The commutators of the supersymmetry transformations for the OCWFs close without use of the wave equations (i.e. the commutators close off-shell).

In Section 8, the global supersymmetry transformations of the TCWFs are derived. The global supersymmetry transformations of the TCWFs involve mass parameters and are different in structure to the chiral and vector multiplets used in standard supersymmetric field theory ${ }^{3,11}$. The commutators of the supersymmetry transformations of the transformed spinor wavefunctions require use of the equations of motion for closure. Auxiliary component wave functions are introduced into the TCWF multiplets that allow closure of the global supersymmetry transformation commutators off-shell. An action is derived for the TCWFs from which the transformed wave equations can be derived. Auxiliary component terms are added to this action to maintain the invariance, up to a total derivative, under the offshell supersymmetry transformations. However, if hermitian conjugation is used in this action for the TCWFs a relative minus sign arises between a supermultiplet representing particles of one mass and the two other supermultiplets representing particles of two other masses. The relative sign in these terms indicates that if the later two supermultiplets represent physical particles than the first supermultiplet will represent ghost particles.

In Section 9, an alternative conjugation is used to define the actions for the OCWFs and TCWFs. The conjugation is based on the Krein space approach to states with indefinite probability. The action remains hermitian but terms from each supermultiplet contribute to the probability with the same sign. A current is defined with no ghost contributions.

\section{The superparticle action}

The following superparticle action ${ }^{5}$, with $\mathrm{N}=1$ global supersymmetry, will be used in this paper:

$$
A=\int_{\tau_{i}}^{\tau_{f}} d \tau\left(m \sqrt{\pi^{a} \pi_{a}+M^{-1} \dot{\bar{\theta}} \dot{\theta}+i N^{-1} \dot{\bar{\theta}} \gamma_{5} \dot{\theta}}\right)
$$


where

$$
\pi^{a}=\dot{x}^{a}-i \bar{\theta}^{\alpha} \gamma_{\alpha}^{a \beta} \dot{\theta}_{\beta}
$$

and $x^{a}$ is a four-vector that represents the even spacetime coordinates, $\theta_{\alpha}$ is a Majorana spinor $\left(\bar{\theta}^{\alpha}=C^{\alpha \beta} \theta_{\beta}\right.$, where $C^{\alpha \beta}$ is the inverse charge conjugation matrix) that represents the four odd Grassmann coordinates ${ }^{3,11}, \gamma_{a \alpha}^{\beta}$ are the Dirac matrices, $\tau$ represents the length of the particle worldline and $m, M$ and $N$ are mass parameters. The quantisation without the line element terms containing $M$ and $N$ was investigated previously by Casalbuoni ${ }^{1}$. The introduction of the additional term containing $M$ and $N$ in the above action has significant effects in the quantisation procedure.

\section{The superparticle Hamiltonian}

The superparticle action (1) has a single first class constraint ${ }^{2}$ in momentum phase space:

$$
G=\left(P^{a} P_{a}-m^{2}\right)+\bar{M} \bar{D} D+i \bar{N} \bar{D} \gamma_{5} D=0
$$

where

$$
R=\left(\bar{M}^{2}+\bar{N}^{2}\right)^{\frac{1}{2}}, \bar{M}=\frac{M^{-1}}{M^{-2}+N^{-2}}, \bar{N}=\frac{N^{-1}}{M^{-2}+N^{-2}}
$$

and

$$
D_{\alpha}=d_{\alpha}-i(\gamma \cdot P \theta)_{\alpha}
$$

and $P^{a}$ and $d_{\alpha}$ are the conjugate momenta to $x^{a}$ and $\theta_{\alpha}$ and $\gamma \cdot P=\gamma^{a} P_{a}$.

The canonical Hamiltonian is zero and hence the Dirac Generalised Hamiltonian is proportional to the constraint:

$$
H=e\left(\left(P^{a} P_{a}-m^{2}\right)+\bar{M} \bar{D} D+i \bar{N} \bar{D} \gamma_{5} D\right)
$$

where $e(\tau)$ is a Lagrange multiplier ${ }^{2}$. The Poisson brackets are:

$$
\begin{gathered}
\left\{x^{a}, P_{b}\right\}=\delta_{b}^{a} \\
\left\{\bar{\theta}^{\alpha}, d_{\beta}\right\}=\delta_{\beta}^{\alpha} \\
\left\{\bar{d}^{\alpha}, \theta_{\beta}\right\}=-\delta_{\beta}^{\alpha}
\end{gathered}
$$

\section{Operator constraints on superwavefunction}

To perform the canonical quantisation the phase space variables are mapped to the following operators:

$$
\begin{aligned}
x^{a} & \longrightarrow x^{a} \\
P^{a} & \longrightarrow-i \frac{\partial}{\partial x_{a}}=-i \partial^{a} \\
\theta_{\alpha} & \longrightarrow \theta_{\alpha} \\
d_{\alpha} & \longrightarrow+i \frac{\partial}{\partial \bar{\theta}^{\alpha}}
\end{aligned}
$$


The even operators satisfy the commutator:

$$
\left[x^{a}, P_{b}\right]_{-}=i \delta_{b}^{a}
$$

and the odd operators satisfy the anti-commutator:

$$
\left[\bar{\theta}^{\alpha}, d_{\beta}\right]_{+}=i \delta_{\beta}^{\alpha}
$$

On canonical quantisation the constraint (6) becomes an operator acting on a superwavefunction $^{2}$. A scalar superwavefunction is chosen, as the simplest case, to form a supersymmetric generalisation of the Klein Gordon equation:

$$
\left[\begin{array}{l}
-\left(\square+m^{2}\right) \\
+\bar{M}\left(\frac{\partial}{\partial \theta} \frac{\partial}{\partial \theta}+i \bar{\theta} \gamma \cdot \partial \frac{\partial}{\partial \theta}+i \frac{\partial}{\partial \theta} \gamma \cdot \partial \theta-\bar{\theta} \theta \square\right) \\
+i \bar{N}\left(\frac{\partial}{\partial \theta} \gamma_{5} \frac{\partial}{\partial \theta}+i \bar{\theta} \gamma \cdot \partial \gamma_{5} \frac{\partial}{\partial \theta}+i \frac{\partial}{\partial \theta} \gamma_{5} \gamma \cdot \partial \theta+\bar{\theta} \gamma_{5} \theta \square\right)
\end{array}\right] \Phi=0
$$

where $\square=\partial^{a} \partial_{a}$.

In this paper the superspace spinor coordinates have one supersymmetry $(N=1)$ and hence no internal symmetry group ${ }^{3,11}$. The expansion of the superwavefunction contains two Dirac spinors, four complex scalars and a complex vector:

$$
\begin{aligned}
\Phi= & f+R^{1 / 2} \bar{\theta} \tilde{\phi}+R \bar{\theta} \theta \tilde{g}+R \bar{\theta} \gamma_{5} \theta \tilde{h} \\
& +R \bar{\theta} \gamma^{m} \gamma_{5} \theta v_{m}+R^{3 / 2} \bar{\theta} \theta \bar{\theta} \tilde{\chi}+R^{2} \bar{\theta} \theta \bar{\theta} \theta k
\end{aligned}
$$

To simplify the wave equations, the additional mass parameter $R$ is used above to scale the component wavefunctions in order that they have the same mass magnitude.

\section{Component wavefunction equations of motion}

To simplify the equations of motion of the component wavefunctions the following redefinitions of the component wavefunctions $\tilde{\chi}, \tilde{\phi}, \tilde{g}$ and $\tilde{h}$ are made

$$
\begin{array}{r}
\chi=\Lambda_{+}^{(1)} \tilde{\chi} \\
\phi=\Lambda_{+}^{(2)} \tilde{\phi} \\
g=\left(\bar{M} R^{-1} \tilde{g}+i \bar{N} R^{-1} \tilde{h}\right) \\
h=\left(i \bar{N} R^{-1} \tilde{g}+\bar{M} R^{-1} \tilde{h}\right)
\end{array}
$$

where

$$
\begin{aligned}
\Lambda_{ \pm}^{(1)} & =(2 R)^{-1}\left[(R+\bar{M} \pm i \bar{N})+\gamma_{5}(R-\bar{M} \mp i \bar{N})\right] \\
\Lambda_{ \pm}^{(2)} & =(2 R)^{-1}\left[(R+\bar{M} \pm i \bar{N})-\gamma_{5}(R-\bar{M} \mp i \bar{N})\right]
\end{aligned}
$$

When the constraint (13) is applied to the superwavefunction (14), three sets of coupled equations of motion are produced in an expansion in terms of $\theta^{\alpha}$. A set of scalar wavefunction equations:

$$
-\left(\square+m^{2}\right) f+8 R^{2} g=0
$$


$6 \quad$ P.L.FitzGerald

$$
\begin{gathered}
-\left(\square+m^{2}\right) g-\square f+8 R^{2} k=0 \\
-\left(\square+m^{2}\right) k-\square g=0
\end{gathered}
$$

A set of spinor wavefunction equations:

$$
\begin{gathered}
-\left(\square+m^{2}\right) \phi_{\alpha}+4 R^{2} \chi_{\alpha}+2 R i(\gamma \cdot \partial \phi)_{\alpha}=0 \\
-\left(\square+m^{2}\right) \chi_{\alpha}-\square \phi_{\alpha}+2 R i(\gamma \cdot \partial \chi)_{\alpha}=0
\end{gathered}
$$

A set of vector-scalar wavefunction equations:

$$
\begin{aligned}
& -\left(\square+m^{2}\right) h+4 R i \partial \cdot v=0 \\
& -\left(\square+m^{2}\right) v_{b}+4 R i \partial_{b} h=0
\end{aligned}
$$

\section{Solution of the equations of motion}

It was shown in Section 5 that the OCWFs in the superwavefunction $\Phi$ (14) do not satisfy wave equations that are suitable to describe the known types of particles. However, three sets of wave equations (17-21) can be diagonalised by the following transformations. The transformation for scalar wave equations (17-19) involves purely algebraic parameters:

$$
\begin{gathered}
g^{(\alpha)}=\left(-\frac{m^{2}}{\left(\alpha^{2}-\beta^{2}\right)} f+\frac{1}{2} g-\frac{2 R}{(\alpha-\beta)} k\right) N_{(\alpha)} \\
g^{(\beta)}=\left(\frac{m^{2}}{\left(\alpha^{2}-\beta^{2}\right)} f+\frac{1}{2} g+\frac{2 R}{(\alpha-\beta)} k\right) N_{(\beta)} \\
g^{(m)}=2^{\frac{1}{2}}\left(-\frac{m}{4 R} f+\frac{2 R}{m} g+\frac{2 R}{m} k\right) N_{(m)}
\end{gathered}
$$

where the masses $\alpha$ and $(-\beta)$ are both positive

$$
\begin{aligned}
& \alpha=2 R+\sqrt{4 R^{2}+m^{2}} \\
& \beta=2 R-\sqrt{4 R^{2}+m^{2}}
\end{aligned}
$$

and

$$
\begin{gathered}
N_{(m)}=\sqrt{2} \\
N_{(\alpha)}=\sqrt{\frac{m^{2}}{\alpha^{2}}+1} \\
N_{(\beta)}=\sqrt{\frac{m^{2}}{\beta^{2}}+1}
\end{gathered}
$$

Substituting (24-26) into (17-19) produces the required Klein-Gordon equations for the transformed scalar wavefunctions (24-26):

$$
\left(\square+\alpha^{2}\right) g^{(\alpha)}=0
$$


The Superfield Quantisation of a Superparticle Action with an Extended Line Element 7

$$
\begin{gathered}
\left(\square+\beta^{2}\right) g^{(\beta)}=0 \\
\left(\square+m^{2}\right) g^{(m)}=0
\end{gathered}
$$

The transformation that will linearise the vector-scalar wavefunction equations (22-23) involves parameters containing spacetime derivatives:

$$
\begin{gathered}
B_{n}^{(m)}=2^{\frac{1}{2}}\left(v_{n}-i \frac{4 R}{m^{2}} \partial_{n} h+m^{-2} \partial_{n} \partial \cdot v\right) N_{(m)} \\
b^{(\alpha)}=\frac{\beta}{(\alpha-\beta)}\left(h+i \beta^{-1} \partial \cdot v\right) N_{(\alpha)} \\
b^{(\beta)}=\frac{\alpha}{(\beta-\alpha)}\left(h+i \alpha^{-1} \partial \cdot v\right) N_{(\beta)}
\end{gathered}
$$

The transformed scalars $b^{(\alpha)}$ and $b^{(\beta)}$ satisfy the second order Klein-Gordon equations, while that of the wavefunction $B_{n}^{(m)}$ satisfies the Proca equation for a massive vector particle:

$$
\begin{gathered}
\left(\square+\alpha^{2}\right) b^{(\alpha)} \\
\left(\square+\beta^{2}\right) b^{(\beta)} \\
\square B_{n}^{(m)}-\partial_{n} \partial \cdot B^{(m)}+m^{2} B_{n}^{(m)}=0
\end{gathered}
$$

The vector-scalar set of equations (22-23) have five degrees of freedom off-shell and on-shell represented by $v_{m}$ and $h$, and five degrees of freedom on-shell represented by $B_{q}^{(m)}, b^{(\alpha)}$ and $b^{(\beta)}$. The transformation (35-37) is therefore only valid on-shell in the TCWFs. The off-shell theory for the TCWFs will be completed in Section 8.

The wavefunctions $\phi_{\alpha}$ and $\chi_{\alpha}$ are Dirac spinors with four off-shell complex components each. The second order equations of motion that they satisfy (20-21) imply that they also each have four on-shell complex components. Spinor wavefunctions that satisfy first order Dirac equations of motion have four complex components offshell and two complex components on-shell. A transformation is therefore required that will map the wavefunctions $\phi_{\alpha}$ and $\chi_{\alpha}$ onto four on-shell spinor wavefunctions satisfying Dirac equations. Building on the procedure for the the scalar and vector transformations, linear combinations of the $\phi_{\alpha}$ and $\chi_{\alpha}$ equations of motion are taken to produce new wavefunctions that satisfy Dirac equations with masses equal to $m, \alpha$ and $\beta$. The transformation parameters involve spacetime derivatives. As in the case for the vector-scalar wavefunctions, the transformation of the spinors is only valid on-shell for the TCWFs.

The transformations for the spinor wave functions are:

$$
\begin{aligned}
& \left(\begin{array}{c}
\rho^{(m+)} \\
\rho^{(m-)}
\end{array}\right)=(8 R m)^{-1} R^{\frac{1}{2}} N_{(m)} \\
& \cdot\left(\begin{array}{c}
(2 R+m) i \gamma \cdot \partial-m^{2}, 2 R i \gamma \cdot \partial+2 R(2 R-m) \\
(2 R-m) i \gamma \cdot \partial-m^{2}, 2 R i \gamma \cdot \partial+2 R(2 R+m)
\end{array}\right)\left(\begin{array}{l}
\phi \\
\chi
\end{array}\right)
\end{aligned}
$$


and

$$
\begin{gathered}
\left(\begin{array}{c}
\rho^{(\beta)} \\
\rho^{(\alpha)}
\end{array}\right)=\left(\begin{array}{cc}
(4 R(\alpha-\beta))^{-1} R^{\frac{1}{2}} N_{(\alpha)}, & 0 \\
0 & ,(4 R(\beta-\alpha))^{-1} R^{\frac{1}{2}} N_{(\beta)}
\end{array}\right) \\
\cdot\left(\begin{array}{c}
(2 R-\beta) i \gamma \cdot \partial+m^{2}, 2 R i \gamma \cdot \partial+2 R(2 R-\beta) \\
(2 R-\alpha) i \gamma \cdot \partial+m^{2}, 2 R i \gamma \cdot \partial+2 R(2 R-\alpha)
\end{array}\right)\left(\begin{array}{c}
\phi \\
\chi
\end{array}\right)
\end{gathered}
$$

The transformed spinor wavefunctions satisfy the following equations of motion:

$$
\begin{gathered}
(i \gamma \cdot \partial+m) \rho^{(m+)}=0 \\
(i \gamma \cdot \partial-m) \rho^{(m-)}=0 \\
(i \gamma \cdot \partial+\beta) \rho^{(\beta)}=0 \\
(i \gamma \cdot \partial+\alpha) \rho^{(\alpha)}=0
\end{gathered}
$$

The wavefunctions $\rho_{(5)}^{(\alpha)}=\gamma_{5} \rho^{(\alpha)}, \rho^{(\beta)}, \rho_{(5)}^{(m+)}=\gamma_{5} \rho^{(m+)}$ and $\rho^{(m-)}$ satisfy positive mass Dirac equations and are the wavefunctions that represent physical particles.

The transformation of the OCWFs to the new TCWFs, that satisfy KleinGordon, Dirac and massive vector equations of motion, is now complete. Three supersymmetric multiplets have been derived: a multiplet with mass $m$ containing one complex vector, two Dirac spinors and one complex scalar; two multiplets containing a Dirac spinor and two complex scalars, one with mass $\alpha$ and one with mass $(-\beta)$. Similar multiplets of Fock space states were derived using a Fock space approach $^{5}$. Note that the supersymmetry transformations of the TCWFs that will be derived in Section 8 require Dirac and not Majorana spinors.

\section{Supersymmetry transformations, commutators and invariant action for the OCWFs}

The transformations derived in Section 6 map the OCWFs to three on-shell supersymmetric multiplets of TCWFs, containing eight bosonic and eight fermionic complex degrees of freedom. The number of off-shell complex degrees of freedom for the TCWFs are nine bosonic and sixteen fermionic. This difference in degrees of freedom suggests that auxiliary wavefunctions are required to form off-shell supersymmetric multiplets for the $\mathrm{TCWFs}^{3}$. The auxiliary wavefunctions can be obtained by ensuring the closure of supersymmetry transformation commutators of the TCWFs off-shell. In this Section, as a preliminary step, the supersymmetry transformations of the OCWFs will be derived.

The supersymmetry transformations are generated by the action of the supersymmetric charge operator $\hat{Q}_{\alpha}$ on the superwavefunction:

$$
\delta_{\varepsilon} \Phi=\bar{\varepsilon}^{\alpha} \hat{Q}_{\alpha} \Phi=\left(i \bar{\varepsilon} \frac{\partial}{\partial \bar{\theta}}-\bar{\varepsilon} \gamma \cdot \partial \theta\right) \Phi
$$

The OCWFs transformations are:

$$
\delta_{\varepsilon} f=i R^{\frac{1}{2}} \bar{\zeta} \phi
$$


The Superfield Quantisation of a Superparticle Action with an Extended Line Element 9

$$
\begin{gathered}
\delta_{\varepsilon} g=-\frac{1}{4} R^{-\frac{1}{2}}(\bar{\zeta} \gamma \cdot \partial \phi)+\frac{i}{2} R^{\frac{1}{2}}(\bar{\zeta} \chi) \\
\delta_{\varepsilon} k=-\frac{1}{4} R^{-\frac{1}{2}}(\bar{\zeta} \gamma \cdot \partial \chi) \\
\delta_{\varepsilon} h=-\frac{1}{4} R^{-\frac{1}{2}}\left(\bar{\zeta} \gamma \cdot \partial \gamma_{5} \phi\right)-\frac{i}{2} R^{\frac{1}{2}}\left(\bar{\zeta} \gamma_{5} \chi\right) \\
\delta_{\varepsilon} v_{a}=\frac{1}{4} R^{-\frac{1}{2}}\left(\bar{\zeta} \gamma \cdot \partial \gamma_{a} \gamma_{5} \phi\right)+\frac{i}{2} R^{\frac{1}{2}}\left(\bar{\zeta} \gamma_{a} \gamma_{5} \chi\right) \\
\delta_{\varepsilon} \phi_{\alpha}=-R^{-\frac{1}{2}}(\gamma \cdot \partial \zeta)_{\alpha} f+2 i R^{\frac{1}{2}}(\zeta)_{\alpha} g \\
+2 i R^{\frac{1}{2}}\left(\gamma_{5} \zeta\right)_{\alpha} h+2 i R^{\frac{1}{2}}\left(\gamma^{b} \gamma_{5} \zeta\right)_{\alpha} v_{b} \\
\delta_{\varepsilon} \chi_{\alpha}=-R^{-\frac{1}{2}}(\gamma \cdot \partial \zeta)_{\alpha} g+4 i R^{\frac{1}{2}}(\zeta)_{\alpha} k \\
-R^{-\frac{1}{2}}\left(\gamma \cdot \partial \gamma_{5} \zeta\right)_{\alpha} h-R^{-\frac{1}{2}}\left(\gamma^{b} \gamma \cdot \partial \gamma_{5} \zeta\right)_{\alpha} v_{b}
\end{gathered}
$$

where $\zeta=\Lambda_{+}^{(1)} \varepsilon$. The commutators of the supersymmetry transformations OCWFs close onto a spacetime translation on- and off-shell, e.g:

$$
\left[\delta_{\varepsilon_{2}}, \delta_{\varepsilon_{1}}\right] f=2 i\left(\bar{\varepsilon}_{1} \gamma^{a} \varepsilon_{2}\right) \partial_{a} f
$$

The equations of motion for the OCWFs are derivable from the following action:

$$
A_{O C W F}=\int d^{4} x d^{4} \theta\left(-\partial^{a} \bar{\Phi} \partial_{a} \Phi-m^{2} \bar{\Phi} \Phi+M \bar{D}^{\alpha} \bar{\Phi} D_{\alpha} \Phi+i N \bar{D}^{\alpha}\left(\gamma_{5}\right)_{\alpha}^{\beta} \bar{\Phi} D_{\beta} \Phi\right)
$$

The only terms that contribute to the action (56) are those of order $\bar{\theta} \theta \bar{\theta} \theta$. After performing the $\theta$ integral the action reduces to:

$$
\begin{aligned}
A_{O C W F}= & R^{2} \int d^{4} x\left(\partial^{a} f^{K} \partial_{a} k+\partial^{a} k^{K} \partial_{a} f+\partial^{a} f^{K} \partial_{a} g+\partial^{a} g^{K} \partial_{a} f\right. \\
& +\partial^{a} g^{K} \partial_{a} g+\partial^{a} h^{K} \partial_{a} h+\partial^{a} v^{b K} \partial_{a} v_{b} \\
& +\frac{1}{4}\left(\partial^{a} \bar{\phi} \partial_{a} \chi+\partial^{a} \bar{\chi} \partial_{a} \phi+\partial^{a} \bar{\phi} \partial_{a} \phi\right) \\
& -m^{2}\left(f^{K} k+k^{K} f+g^{K} g+h^{K} h+v^{a K} v_{a}+\frac{1}{4} \bar{\phi} \chi+\frac{1}{4} \bar{\chi} \phi\right) \\
& +R^{2}\left(8 g^{K} k+8 k^{K} g+\bar{\chi} \chi\right)+i 4 R\left(v^{a K} \partial_{a} h-\partial_{a} h^{K} v^{a}\right) \\
& \left.+i \frac{R}{4}\left(\bar{\phi} \gamma \cdot \partial \chi-\partial_{a} \bar{\chi} \gamma^{a} \phi+\bar{\chi} \gamma \cdot \partial \phi-\partial_{a} \bar{\phi} \gamma^{a} \chi\right)\right)
\end{aligned}
$$

The type of conjugation is not yet fixed. In (57), $f^{K}$ is the conjugate variable to $f$ and $\bar{\chi}=\chi^{K} A$ where $A \gamma^{a} A^{-1}=\gamma^{\dagger}$. Supersymmetry transformations of the OCWFs transforms the Lagrangian to a total derivative. 


\section{Supersymmetry transformations, commutators and invariant action for the TCWFs}

Using (48-54) the following supersymmetry transformations can be derived for the TCWFs:

$$
\begin{gathered}
\delta_{\varepsilon} g^{(\alpha)}=i\left(\bar{\zeta} \rho^{(\alpha)}\right) \\
\delta_{\varepsilon} g^{(\beta)}=i\left(\bar{\zeta} \rho^{(\beta)}\right) \\
\delta_{\varepsilon} g^{(m)}=i 2^{-\frac{1}{2}}\left(\bar{\zeta}\left(\rho^{(m+)}+\rho^{(m-)}\right)\right) \\
\delta_{\varepsilon} b^{(\alpha)}=i\left(\bar{\zeta} \gamma_{5} \rho^{(\alpha)}\right) \\
\delta_{\varepsilon} b^{(\beta)}=i\left(\bar{\zeta} \gamma_{5} \rho^{(\beta)}\right) \\
\delta_{\varepsilon} \rho_{\alpha}^{(\alpha)}=i\left[((i \gamma \cdot \partial-\alpha) \zeta)_{\alpha} g^{(\alpha)}-\left((i \gamma \cdot \partial-\alpha) \gamma_{5} \zeta\right)_{\alpha} b^{(\alpha)}\right] \\
\delta_{\varepsilon} \rho_{\alpha}^{(\beta)}=i\left[((i \gamma \cdot \partial-\beta) \zeta)_{\alpha} g^{(\beta)}-\left((i \gamma \cdot \partial-\beta) \gamma_{5} \zeta\right)_{\alpha} b^{(\beta)}\right] \\
\delta_{\varepsilon} B_{n}^{(m)}=i 2^{-\frac{1}{2}}\left(\bar{\zeta} \gamma_{5} \gamma_{n}\left(\rho^{(m+)}-\rho^{(m-)}\right)\right) \\
-m^{-1}\left(\bar{\zeta} \gamma_{5}\left(\partial_{n} \rho^{m+}+\partial_{n} \rho^{m-}\right)\right) \\
\delta_{\varepsilon} \rho_{\alpha}^{(m+)}=i 2^{-\frac{1}{2}}\left[((i \gamma \cdot \partial-m) \zeta)_{\alpha} g^{(m)}\right. \\
\left.+\left(\left(\sigma^{m n} \partial_{m} B_{n}^{(m)}-m \gamma^{n} B_{n}^{(m)}\right) \gamma_{5} \zeta\right)_{\alpha}\right] \\
\delta_{\varepsilon} \rho_{\alpha}^{(m-)}=i 2^{-\frac{1}{2}}\left[((i \gamma \cdot \partial+m) \zeta)_{\alpha} g^{(m)}\right. \\
\left.-\left(\left(\sigma^{m n} \partial_{m} B_{n}^{(m)}+m \gamma^{n} B_{n}^{(m)}\right) \gamma_{5} \zeta\right)_{\alpha}\right]
\end{gathered}
$$

In generating the supersymmetry transformations of the TCWFs from those of the OCWFs (48-54) the equations of motion (32-34), (38-40), (44) and (46) are set to zero and the above transformations are therefore on-shell.

The supersymmetric transformations of the TCWFs (58-67) are novel in that they contain mass parameters and hence cannot be mapped onto those of the chiral and vector multiplets previously used in superfield theories supersymmetric transformations ${ }^{3,11}$.

The commutators of the supersymmetry transformations of the transformed spinor wavefunctions (64-67) contain terms proportional to the equations of motion. These terms can be removed by the addition of seven auxiliary wavefunctions that have the following supersymmetry transformations:

$$
\delta_{\varepsilon} F^{(\alpha)}=i \bar{\zeta}(i \gamma \cdot \partial+\alpha) \rho^{(\alpha)}
$$


The Superfield Quantisation of a Superparticle Action with an Extended Line Element 11

$$
\begin{aligned}
\delta_{\varepsilon} F^{(\beta)} & =i \bar{\zeta}(i \gamma \cdot \partial+\beta) \rho^{(\beta)} \\
\delta_{\varepsilon} F^{(m+)} & =i \bar{\zeta}(i \gamma \cdot \partial+m) \rho^{(m+)} \\
\delta_{\varepsilon} F^{(m-)} & =i \bar{\zeta}(i \gamma \cdot \partial-m) \rho^{(m-)} \\
\delta_{\varepsilon} G^{(\alpha)} & =i \bar{\zeta} \gamma_{5}(i \gamma \cdot \partial+\alpha) \rho^{(\alpha)} \\
\delta_{\varepsilon} G^{(\beta)} & =i \bar{\zeta} \gamma_{5}(i \gamma \cdot \partial+\beta) \rho^{(\beta)} \\
\delta_{\varepsilon} G^{(m)}= & i 2^{-\frac{1}{2}} \bar{\zeta} \gamma_{5}(i \gamma \cdot \partial+m) \rho^{(m+)} \\
& +i 2^{-\frac{1}{2}} \bar{\zeta} \gamma_{5}(i \gamma \cdot \partial-m) \rho^{(m-)}
\end{aligned}
$$

and the addition of auxiliary wavefunction terms in the supersymmetry transformations of the spinor wavefunctions (64-67):

$$
\begin{gathered}
\delta_{\varepsilon} \rho_{\alpha}^{(\alpha)} \longrightarrow \delta_{\varepsilon} \rho_{\alpha}^{(\alpha)}+(\zeta)_{\alpha} F^{(\alpha)}-2^{-\frac{1}{2}}\left(\gamma_{5} \zeta\right)_{\alpha} G^{(\alpha)} \\
\delta_{\varepsilon} \rho_{\alpha}^{(\beta)} \longrightarrow \delta_{\varepsilon} \rho_{\alpha}^{(\beta)}+(\zeta)_{\alpha} F^{(\beta)}-2^{-\frac{1}{2}}\left(\gamma_{5} \zeta\right)_{\alpha} G^{(\beta)} \\
\delta_{\varepsilon} \rho_{\alpha}^{(m+)} \longrightarrow \delta_{\varepsilon} \rho_{\alpha}^{(m+)}+(\zeta)_{\alpha} F^{(m+)}-2^{-\frac{1}{2}}\left(\gamma_{5} \zeta\right)_{\alpha} G^{(m)} \\
\delta_{\varepsilon} \rho_{\alpha}^{(m-)} \longrightarrow \delta_{\varepsilon} \rho_{\alpha}^{(m-)}+(\zeta)_{\alpha} F^{(m-)}-2^{-\frac{1}{2}}\left(\gamma_{5} \zeta\right)_{\alpha} G^{(m)}
\end{gathered}
$$

With the inclusion of the auxiliary wavefunctions the commutators of the supersymmetry transformations for the TCWFs close off-shell.

The equations of motion of the TCWFs (32-34), (38-40), (44) and (46) are derivable from the following action for massive scalar, spinor and vector particles:

$$
\begin{aligned}
A_{t c w f}= & \int d^{4} x\left(S _ { ( m ) } \left[\partial^{a} g^{(m) K} \partial_{a} g^{(m)}-m^{2} g^{(m) K} g^{(m)}\right.\right. \\
& +\frac{1}{2}\left(\partial_{a} B_{b}^{(m) K}-\partial_{b} B_{a}^{(m) K}\right)\left(\partial^{a} B^{(m) b}-\partial^{b} B^{(m) a}\right)-m^{2} B_{a}^{(m) K} B^{(m) a} \\
& +\frac{i}{2} \bar{\rho}_{(5)}^{(m+)} \gamma \cdot \partial \rho_{(5)}^{(m+)}-\frac{i}{2} \partial_{a} \bar{\rho}_{(5)}^{(m+)} \gamma^{a} \rho_{(5)}^{(m+)}-m \bar{\rho}_{(5)}^{(m+)} \rho_{(5)}^{(m+)} \\
& \left.+\frac{i}{2} \bar{\rho}^{(m-)} \gamma \cdot \partial \rho^{(m-)}-\frac{i}{2} \partial_{a} \bar{\rho}^{(m-)} \gamma^{a} \rho^{(m-)}-m \bar{\rho}^{(m-)} \rho^{(m-)}\right] \\
& +S_{(\alpha)}\left[\partial^{a} g^{(\alpha) K} \partial_{a} g^{(\alpha)}-\alpha^{2} g^{(\alpha) K} g^{(\alpha)}+\partial^{a} b^{(\alpha) K} \partial_{a} b^{(\alpha)}-\alpha^{2} b^{(\alpha) K} b^{(\alpha)}\right. \\
& \left.+\frac{i}{2} \bar{\rho}_{(5)}^{(\alpha)} \gamma \cdot \partial \rho_{(5)}^{(\alpha)}-\frac{i}{2} \partial_{a} \bar{\rho}_{(5)}^{(\alpha)} \gamma^{a} \rho_{(5)}^{(\alpha)}-\alpha \bar{\rho}_{(5)}^{(\alpha)} \rho_{(5)}^{(\beta)}\right] \\
& +S_{(\beta)}\left[\partial^{a} g^{(\beta) K} \partial_{a} g^{(\beta)}-\beta^{2} g^{(\beta) K} g^{(\beta)}+\partial^{a} b^{(\beta) K} \partial_{a} b^{(\beta)}-\alpha^{2} b^{(\beta) K} b^{(\beta)}\right. \\
& \left.\left.+\frac{i}{2} \bar{\rho}^{(\beta)} \gamma \cdot \partial \rho^{(\beta)}-\frac{i}{2} \partial_{a} \bar{\rho}^{(\beta)} \gamma^{a} \rho^{(\beta)}+\beta \bar{\rho}^{(\beta)} \rho^{(\beta)}\right]\right)
\end{aligned}
$$

where $S_{(m)}$ is equal to -1 and $S_{(\alpha)}$ and $S_{(\beta)}$ are equal to +1 . The action is invariant under the on-shell TCWF supersymmetry transformations (58-67) (the Lagrangian transforms to a total derivative). The TCWF action can be made invariant to the 
off-shell supersymmetry transformations (68-78) by the addition of the following terms containing the auxiliary wavefunctions:

$$
\begin{aligned}
A_{t c w f} \longrightarrow & A_{t c w f}- \\
& \int d^{4} x\left(S_{(m)}\left[F^{K(m+)} F^{(m+)}+F^{K(m-)} F^{(m-)}+G^{K(m)} G^{(m)}\right]\right. \\
& +S_{(\alpha)}\left[F^{K(\alpha)} F^{(\alpha)}+G^{K(\alpha)} G^{(\alpha)}\right]+S_{(\beta)}\left[F^{K(\beta)} F^{(\beta)}+G^{K(\beta)} F^{(\beta)}\right](\$ 0)
\end{aligned}
$$

where $S_{(m)}$ is equal to -1 and $S_{(\alpha)}$ and $S_{(\beta)}$ are equal to +1 . Unlike other examples of off-shell supersymmetric actions ${ }^{3,11}$ the auxiliary wavefunctions are completely decoupled from the on-shell wavefunctions.

A conserved current (i.e. conserved when the equations of motion are satisfied) can be derived from the OCWF action that transforms into a conserved current for the TCWF action.

$$
\begin{aligned}
j_{a}= & S_{(m)} i\left[\partial_{a} g^{(m) K} g^{(m)}-g^{(m) K} \partial_{a} g^{(m)}\right. \\
& +\partial_{a} B_{b}^{(m) K} B^{(m) b}-B_{b}^{(m) K} \partial_{a} B^{(m) b} \\
& +\frac{i}{2} \bar{\rho}_{(5)}^{(m+)} \gamma_{a} \rho_{(5)}^{(m+)} \\
& \left.+\frac{i}{2} \bar{\rho}^{(m-)} \gamma_{a} \rho^{(m-)}\right] \\
& +S_{(\alpha)} i\left[\partial^{a} g^{(\alpha) K} g^{(\alpha)}-g^{(\alpha) K} \partial_{a} g^{(\alpha)}+\partial^{a} b^{(\alpha) K} b^{(\alpha)}-b^{(\alpha) K} \partial_{a} b^{(\alpha)}\right. \\
& \left.+\frac{i}{2} \bar{\rho}_{(5)}^{(\alpha)} \gamma_{a} \rho_{(5)}^{(\alpha)}\right] \\
& +S_{(\beta)} i\left[\partial^{a} g^{(\beta) K} g^{(\beta)}-g^{(\beta) K} \partial_{a} g^{(\beta)}+\partial^{a} b^{(\beta) K} b^{(\beta)}-b^{(\beta) K} \partial_{a} b^{(\beta)}\right. \\
& \left.\left.+\frac{i}{2} \bar{\rho}^{(\beta)} \gamma_{a} \rho^{(\beta)}\right]\right)
\end{aligned}
$$

The conserved probability for the superwavefunction (14) is the Lorentz invariant surface integral of this conserved current.

However, there is a relative minus sign in front of the terms for the multiplet of mass $m$ (as $\left.S_{(m)}=-1\right)$ in the TCWF action $(79,80)$ and the current $(81)$, compared with the terms for the other two multiplets of masses $\alpha$ and $(-\beta)$. The relative minus sign will force the TCWF terms in the probability with mass $m$ to be negative ${ }^{12,13}$ when the corresponding TCWF terms arising from the supermultiplets with mass $\alpha$ and $\beta$ are positive.

This problem due to the extended supersymmetric line element in the action 1) is in addition to the inherent problems of positive definite probabilities and negative energy states found in the relativistic component wavefunctions. The later problems are overcome by developing a quantum field theory based on the form of this action using the standard approach for constructing many particle states satisfying the Klein-Gordon, Dirac and Proca equations of motion ${ }^{14}$. However, problem of the relative minus sign in front of the terms in the TCWF action (79) and $(80)\left(S_{(m)}=\right.$ -1 ) for the supermultiplet of mass $m$ will re-emerge in the quantun field theory 
and force the Fock states arising from the fields with mass $m$ to have negative norm ${ }^{12,13,15}$ if the Fock states of equivalent spin arising from the fields with mass $\alpha$ and $\beta$ have positive norm. The existence of ghost states for this multiplet agrees with the result obtained by Volkov and Pashnev using the Fock space approach for first quantisation ${ }^{5}$.

In the next section an alternative conjugation is proposed for the component fields in the actions $(57),(56)$ and $(79,80)$. The action that results will be hermitian but will not have the relative minus sign between the terms for the multiplet of mass $m$ and the multiplets of masses $\alpha$ and $(-\beta)$ and hence contain no ghost states.

\section{Removal of ghosts}

In this section the problem with the relative minus sign between the supersymmetric multiplets in the TCWF action $(79,80)$ is addressed. It will be assumed that the Klein-Gordon, Dirac and Proca equations satisfied by each TCWF within each supermultiplet are treated in the standard way for one particle relativistic quantum mechanics ${ }^{16}$. There will be problems with the interpretation and interactions of the positive and negative energy states and the definition of a positive definite probability ${ }^{16,17}$ for the individual TCWFs. However, these problems need not be addressed here, as there is a meaningful interpretation for each TCWF in a many body theory, based on the Feynmann-Stueckelberg interpretation in quantum field theory ${ }^{14}$ or in the propagator approach for quantum mechanical scattering ${ }^{16}$.

In this section, a solution based on a Krein space approach is used to assign a new conjugation for the TCWFs. Under this Krein space conjugation the actions (57) and $(79,80)$ will still be hermitian but all terms will be positive.

A Krein space approach for quantum mechanics in an indefinite space ${ }^{18,19,20}$ can be used if the set of wavefunctions $F$ can be divided into two sets of wavefunctions, $F_{+}$and $F_{-}$, which have positive definite norm $\left\langle F_{+} \mid F_{+}\right\rangle>0$ and negative definite norm $\left\langle F_{-} \mid F_{-}\right\rangle<0$. If projectors can be defined for the wavefunctions in each space such that $P_{+} F=F_{+}$and $P_{-} F=F_{-}$then a positive definite norm can be formed for the complete set of wavefunctions $\langle F \mid J F\rangle>0$ using the gram operator $J=P_{+}-P_{-}$. In order to produce a positive definite scalar products in spaces of supersymmetric functions a similar construction has been found to be required ${ }^{20,21,22}$ (in this paper we are concerned with the probability current density.) Another interpretation of this procedure is to define a Krein conjugate $F^{K}=(J F)^{\dagger}$ and a rewrite the positive definite norm as $\left\langle F^{K} \mid F\right\rangle$. The replacement of hermitian conjugation in an action is not new. An alternative conjugation, $\bar{\psi}=\psi^{\dagger} A$, is required in order to define a positive definite probability for the Dirac spinor ${ }^{14,16}$.

In this paper, a Krein conjugate will be defined to ensure that the contribution from each TCWF term in the conserved current (81) remains has a positive sign.

Two component spinor notation based on Wess and Bagger ${ }^{3}$, but with a metric 
signiture $(+,-,-,-)$, will be used in this section:

$$
\begin{array}{r}
D_{\alpha}=\left(\begin{array}{c}
D_{\alpha} \\
\bar{D}^{\dot{\alpha}}
\end{array}\right) \\
\gamma_{\alpha}^{\beta}=\left(\begin{array}{cc}
0 & \sigma_{\alpha \dot{\beta}} \\
\bar{\sigma}^{\dot{\alpha} \beta} & 0
\end{array}\right)
\end{array}
$$

The standard projectors for superwavefunctions are ${ }^{3,20}$ :

$$
\begin{gathered}
P_{(1)}=\frac{D^{2} \bar{D}^{2}}{16(-\square)} \\
P_{(2)}=\frac{\bar{D}^{2} D^{2}}{16(-\square)} \\
P_{(T)}=-\frac{D \bar{D}^{2} D}{8(-\square)} \\
P_{(+)}=\frac{D^{2}}{4 \sqrt{(-\square)}} \\
P_{(-)}=\frac{\bar{D}^{2}}{4 \sqrt{(-\square)}}
\end{gathered}
$$

The following projectors, based on the standard projectors,

$$
\begin{aligned}
& Z_{(1)}=P_{(1)}+P_{(2)}+R^{-1}(\bar{M}+i \bar{N}) P_{(+)}+R^{-1}(\bar{M}-i \bar{N}) P_{(-)} \\
& Z_{(2)}=P_{(1)}+P_{(2)}-R^{-1}(\bar{M}+i \bar{N}) P_{(+)}-R^{-1}(\bar{M}-i \bar{N}) P_{(-)} \\
& Z_{(T)}=P_{(T)} \\
& Z_{(+)}=P_{(1)}-P_{(2)}+R^{-1}(\bar{M}+i \bar{N}) P_{(+)}-R^{-1}(\bar{M}-i \bar{N}) P_{(-)} \\
& Z_{(-)}=P_{(1)}-P_{(2)}-R^{-1}(\bar{M}+i \bar{N}) P_{(+)}+R^{-1}(\bar{M}-i \bar{N}) P_{(-)}
\end{aligned}
$$

will be used to define a Krein conjugate for the superwavefunction (14):

$$
\Psi^{K}=\left(\left(Z_{(1)}+Z_{(2)}-Z_{(T)}\right) \Psi\right)^{\dagger}
$$

and the OCWFs and TCWFs. $Z_{(T)}$ is the projector for wavefunctions of mass $m$ and $\left(Z_{(1)}+Z_{(2)}\right)$ is the projector for wavefunctions of mass $\alpha$ and $(-\beta)$. The Krein conjugate can be used in the action for the superwavefunction (56) and hence the TCWF action $(79,80)$ and the definition of the TCWF current $(81)$. The projections of the constraint equation (3) satisfy:

$$
\begin{aligned}
Z_{(1)} G \Psi=G_{(1)} Z_{(1)} \Psi & =0 \\
Z_{(2)} G \Psi=G_{(2)} Z_{(2)} \Psi & =0 \\
Z_{(T)} G \Psi=G_{(T)} Z_{(T)} & =0
\end{aligned}
$$

where the last equalities hold on-shell and

$$
\begin{aligned}
& G_{(1)}=-\square+4 M \sqrt{(-\square)}-m^{2} \\
& G_{(2)}=-\square-4 M \sqrt{(-\square)}-m^{2} \\
& G_{(T)}=-\square-m^{2}
\end{aligned}
$$


The use of the Krein conjugate (94) sets the Krein conjugate of each TCWF of mass $m$ to be equal to minus the hermitian conjugate. The contribution of each TCWF term in the OCWF action (57), the TCWF action $(79,80)$ and current $(81)$ are therefore all positive. This choice of conjugation removes the ghost contributions.

Constantinescu has found that for general supersymmetric scalar products a Krein space approach is required for positivity ${ }^{20,21,22}$.

A Krein space solution can also be applied to the Fock states used by Volkov and Pashnev ${ }^{5}$. There space of Fock states consists of the direct product of a Hilbert space for the multiplets of mass $\alpha$ and $(-\beta)$ and an anti-Hilbert space for the multiplet of mass $m$ (the ghost states). A positive definite norm for Fock states $F$ that exist in a Krein space can be defined by using the number operator for the ghost states $N_{g}{ }^{12,23}$ :

$$
\left\langle F\left|(-1)^{N_{g}}\right| F\right\rangle>0
$$

\section{Conclusions}

It has been shown that canonical quantisation of action based on an extended line element in $\mathrm{N}=1$ global superspace produces on-shell wavefunctions (TCWFs) that satisfy the Klein-Gordon, Dirac and Proca equations. The wave equations have been derived by a series of transformations on the original component wavefunctions (OCWFs) of a scalar superwavefunction. The OCWFs satisfied equations that arise directly from a single first class constraint operator acting on the superwavefunction.

The derivation confirms the result of Volkov and Pashnev ${ }^{5}$ that three multiplets of different mass are produced. In the approach presented, the off-shell content of the three supersymmetric multiplets consists of the following: a multiplet with mass $m$ containing one complex vector, two Dirac spinors and four complex scalars; two multiplets containing a Dirac spinor and four complex scalars, one that has mass $\alpha$ and one that has mass $(-\beta)$. The off-shell component content of the multiplets is twice that of the original $\mathrm{N}=1$ superwavefunction.

A supersymmetric action has been constructed from which the equations of motion of the TCWFs can be derived. In this action the three supersymmetric mass multiplets are decoupled. If hermitian conjugation is used for the congugate variables in this action a relative minus sign arises between terms in one of the supersymmetric mass multiplets compared with terms in the other two supersymmetric mass multiplets. The states arising from the action with hermitian conjugates correspond to the Fock space states in the Volkov-Pashnev quantisation procedure ${ }^{5}$ in which the states of mass $m$ are ghosts. If a Krein conjugation is used for the congugate variables in this action the ghost states are removed and the standard Hilbert space approach can be applied to the construction of a conserved probability ${ }^{16}$ and quantum field theory ${ }^{14}$.

The global supersymmetry transformations derived for the component wavefunctions that represent physical particles (TCWFs) involve mass parameters. These transformations are novel in that they cannot be mapped onto those of the chiral 
and vector multiplets previously used in superfield theories ${ }^{3,11}$. Off-shell closure of the supersymmetry transformation commutators for the spinor wavefunctions has been shown to require the introduction of auxiliary wavefunctions into all three multiplets. In the supersymmetric action for the TCWFs the auxiliary wavefunctions are decoupled from the on-shell wavefunctions.

The approach presented provides a multi-mass, ghost-free supersymmetric free particle model and the foundation for further investigation of an interacting model, based on the extended worldline superparticle action but including gauge potentials.

\section{References}

1. R. Casalbuoni, Il Nuovo. Cimento 33A, 389 (1976).

2. K. Sundermeyer, Constrained dynamics. Lecture Notes in Physics, Vol. 169, (SpringerVerlag, Berlin, Heidelberg, New York, 1982).

3. J. Wess, J. Bagger, Supersymmetry and supergravity, 2nd edition, (Princeton University Press, New Jersey, 1992).

4. A. Frydryszak, Lagrangian models of particles with spin: the first seventy years Preprint hep-th/9601020 (1996).

5. D. V. Volkov and A. T. Pashnev, Teor. Mat. Fiz. 44, 321(1980).

6. B. DeWitt, Supermanifolds, Second Edition, (Cambridge University Press, Cambridge, 1992).

7. A. de Azcarraga, J. Lukierski, Z. Phys. C-Particles and Fields 30, 221 (1986).

8. P. L. FitzGerald, Z. Phys. C-Particles and Fields 44, 313 (1989).

9. A. de Azcarraga, J. Lukierski, Phys. Lett. B 113, 170 (1982).

10. M. Sohnius, Nucl. Phys. B 138, 109 (1978).

11. M. Sohnius, Phys. Rep. 128, 39 (1985).

12. A. van Tonder, Nucl. Phys. B 645, 371 (2002).

13. S. W. Hawking and T. Hertog, Phys. Rev. D 65, 1035151 (2002).

14. J. D. Bjorken and S. D. Drell, Relativistic Quantum Field Theory, (McGraw-Hill, Inc, New York 1964).

15. S. Arnone, Y. A. Kubyshin, T. R. Morris, and J. F. Tighe, Int.J.Mod.Phys. A17, 2283 (2002).

16. J. D. Bjorken and S. D. Drell, Relativistic Quantum Mechanics, (McGraw-Hill, Inc, New York 1964)

17. A. Mostafazadeh Class. Quantum Grav. 20155 (2003).

18. G. S. Japaridze, J. Phys. A: Math. Gen. 35, 1709 (2002).

19. T. Ya. Azizov and I. S. Iokhvidov, 1989 Linear Operators in Spaces with an Indefinite Metric, (New York: Wiley-Interscience 1989).

20. F. Constantinescu, Krein structure of supersymmetry Preprint hep-th/0305143 (16 May 2003).

21. F. Constantinescu, Lett. Math. Phys. 62, 111 (2002).

22. F. Constantinescu, Supersymmetric Kallen-Lehmann representation Preprint hepth/0306019 (June 2003).

23. J-P. Gazeau, J. Renaud and M. V. Takook Class. Quantum Grav. 171415 (2000). 